\title{
Emotional symptoms and sensation seeking: Implications for tobacco interventions for youth and young adults
}

\author{
Bara S. Bataineh', Anna V. Wilkinson², Kathleen R. Case³, Stephanie L. Clendennen², Aslesha Sumbe², Baojiang Chen ${ }^{4}$,
} Melissa B. Harrell ${ }^{2}$

\begin{abstract}
INTRODUCTION Although emotional symptoms and sensation seeking are recognized as important risk factors for tobacco use among youth and young adults, to date, their joint influence on tobacco use has not been examined.

METHOds Data used in this study are from the Texas Adolescent Tobacco and Marketing Surveillance study, a population-based cohort. At baseline, in 2014, participants were in the 10th grade. Mixed-effects logistic regression models examined associations between emotional symptoms and sensation seeking in 2014 and odds of past 30-day cigarette and e-cigarette use in 2018. Interactions between emotional symptoms and sensation seeking were examined to assess whether one modifies the effect of the other on cigarette and e-cigarette use. RESULTS After adjusting for age, sex, race/ethnicity, school type, and ever use of cigarettes or e-cigarettes at baseline, adolescents with high emotional symptoms $\left(\mathrm{AOR}_{\mathrm{cig}}=1.97 ; 95 \% \mathrm{CI}: 1.07-3.60\right.$, and $\left.\mathrm{AOR}_{\mathrm{e}-\mathrm{cig}}=1.68 ; 95 \% \mathrm{CI}: 1.06-2.66\right)$ and with high sensation seeking tendencies $\left(\mathrm{AOR}_{\mathrm{cig}}=2.05\right.$; $95 \% \mathrm{CI}: 1.03-4.10$, and $\mathrm{AOR}_{\mathrm{e}}$ ${ }_{\mathrm{cig}}=1.68 ; 95 \%$ CI: $1.02-2.76$ ) had significantly higher odds of past 30 -day cigarette and e-cigarette use four years later compared to adolescents with low emotional symptoms and low sensation seeking tendencies. The interaction was significant $(\mathrm{p}=0.01)$ for e-cigarette use only; among low sensation seekers, adolescents who reported high levels of emotional symptoms were at increased risk for past 30day use $\left(\mathrm{AOR}_{\mathrm{e}-\mathrm{cig}}=3.43 ; 95 \% \mathrm{CI}: 1.38-8.51\right)$, and among adolescents with low emotional symptoms, high sensation seekers were at increased for risk for past 30-day use ( $\mathrm{AOR}_{\text {e-cig }}=3.50 ; 95 \%$ CI: 1.54-7.91).

CONCLUSIONS It is important for tobacco use prevention programs to consider both behavioral risk factors - sensation seeking and emotional symptoms - in an integrative way, to target high risk subgroups and thereby increase the efficacy of existing effective intervention strategies in order to curb tobacco use among youth and young adults.
\end{abstract}

\section{AFFILIATION}

1 Department of Epidemiology, Human Genetics and Environmental Sciences, School of Public Health, University of Texas Health Science Center, Dallas, United States 2 Department of Epidemiology, Human Genetics and Environmental Sciences, School of Public Health, University of Texas Health Science Center, Austin, United States 3 Center for Research to Advance Community Health (ReACH), University of Texas Health Science Center, San Antonio, United States 4 Department of Biostatistics and Data Science, School of Public Health, University of Texas Health Science Center, Austin, United States

\section{CORRESPONDENCE TO}

Stephanie L. Clendennen. Department of Epidemiology, Human Genetics and Environmental Sciences, School of Public Health, University of Texas Health Science Center, 1616 Guadalupe, TX 78701, Austin, United States. E-mail: Stepahnie.L.Clendennen@uth.tmc. edu

\section{KEYWORDS}

youth, cigarettes, electronic cigarettes, sensation seeking, young adults, emotional symptoms

Received: 25 November 2020

Revised: 9 February 2021

Accepted: 21 February 2021

\section{INTRODUCTION}

Tobacco use remains the leading cause of preventable death in the United States ${ }^{1}$. Tobacco use typically starts and is established primarily during adolescence, when the developing brain is most susceptible to nicotine addiction $^{1,2}$. Nearly 9 in 10 current cigarette smokers tried their first cigarette by the age of 18 years, and $98 \%$ first tried smoking cigarettes by 26 years ${ }^{2}$. In 2019 , about 6 in 100 high school students $(5.8 \%)$ reported that they smoked cigarettes in the past 30 
days - a decrease from $15.8 \%$ in $2011^{3}$. However, in 2019 more than 1 in 4 high school students $(27.5 \%)$ reported using electronic cigarettes (e-cigarettes) in the past 30 days - an increase from $1.5 \%$ in $2011^{3}$. In 2014, the US Food and Drug Administration (FDA) concluded that e-cigarettes are considered tobacco products because most contain nicotine, which comes from tobacco ${ }^{4}$. Both cigarettes and e-cigarettes have similar negative effects on the body in the shortterm, such as excessive coughing, shortness of breath, and fatigue ${ }^{5,6}$. However, to date, the full breadth of health consequences associated with e-cigarette use is unknown. This includes whether their use over time will result in other long-term health consequences similar in magnitude to the well-established negative effects of long-term cigarette use $\mathrm{e}^{1,5}$. Given the negative health consequences of nicotine and tobacco use and these prevalence rates, preventing tobacco product use among youth remains critical to ending the tobacco epidemic in the United States.

\section{Emotional symptoms and tobacco use}

Emotional symptoms in youth and young adults can manifest as mental health symptoms of depression, anxiety, inability to focus attention, and suicide ideation $^{7}$. Nicotine has both anxiolytic ${ }^{8}$ and antidepressive ${ }^{9}$ qualities; in turn one reason smokers report smoking is to reduce emotional symptoms ${ }^{10}$. For some, smoking is initiated in response to negative emotions ${ }^{10}$ and the perception that smoking is a good way to control negative emotions predicts smoking maintenance and escalation ${ }^{11}$. Indeed, youth and young adults with emotional symptoms report a higher prevalence of nicotine use than their peers who do not report emotional symptoms ${ }^{12-15}$. Currently and overall, less is known about the relationship between emotional symptoms and e-cigarette use compared to cigarette use.

\section{Sensation seeking and tobacco use}

Sensation seeking is a personality trait defined by 'the seeking of varied, novel, complex, and intense sensations and experiences, and the willingness to take risks for the sake of such experience" ${ }^{16}$. Both cross-sectional and longitudinal studies have found that as sensation seeking increases, the risk of negative behaviors such as nicotine and tobacco use, including both cigarettes and e-cigarettes, also increase ${ }^{17,18}$. High sensation seeking youth may try tobacco because it is illegal and may enjoy the arousal and sensations associated with contravening social norms and breaking the law. In addition, the novelty of smoking may be attractive to youth with higher levels of sensation seeking ${ }^{19}$. Thus, e-cigarettes, which entered the market in 2007 and have become increasingly popular among youth, may be particularly appealing to sensation seekers, for their novelty.

\section{Emotional symptoms and sensation seeking}

When examining the influence of family dynamics on substance use, Trujillo et al. ${ }^{20}$ report that it is through an increase in negative emotional symptoms, which in turn augments sensation seeking behaviors, that family conflict serves to increase both intentions to use and substance use itself. In this study, both emotional symptoms and sensation seeking mediated the relationship between family dynamics and both alcohol and marijuana use. However, to date, although emotional symptoms and sensation seeking are recognized as important risk factors for tobacco use, how their joint exposure impacts nicotine and tobacco use risk is unclear. We seek to fill this gap.

\section{Study purpose and aims}

Given that emotional symptoms and sensation seeking independently increase risk for nicotine and tobacco use $\mathrm{e}^{12-15,17-19}$, we hypothesize that youth who experience many emotional symptoms and contemporaneously report as high sensation seekers will be at higher risk for nicotine and tobacco use relative to youth who experience few emotional symptoms and contemporaneously report as low sensation seekers. Of importance, sensation seeking is a modifiable trait ${ }^{21}$, and some adolescents with high sensation seeking tendencies choose safe positive behaviors to meet arousal needs ${ }^{22}$. A better understanding of the sensation seeking trait among youth with emotional symptoms may serve to identify youth at risk of future nicotine and tobacco use and may help to hone prevention strategies. Therefore, this study aimed to examine whether: 1) emotional symptoms at baseline are associated with cigarette and e-cigarette use four years later, after adjusting for sociodemographic characteristics; 2) sensation seeking tendencies at baseline are associated with cigarette and e-cigarette use four years later after 
adjusting for sociodemographic characteristics; 3 ) sensation seeking is a potential effect modifier of the association between emotional symptoms and product use; and 4) emotional symptoms are a potential effect modifier of the association between sensation seeking and product use.

\section{METHODS}

\section{Study design and participants}

The data used in this study were derived from the Texas Adolescent Tobacco and Marketing Surveillance System (TATAMS), a longitudinal surveillance study of e-cigarette and other tobacco use behaviors among a population-based cohort of adolescents living in major metropolitan areas of Texas (Austin, Dallas-Ft. Worth, Houston, San Antonio). Baseline data were collected in the classroom during the 2014-2015 academic year from 3907 students in the 6 th, 8 th, and 10th grades using computerized surveys administered via tablets. After wave 2 , all surveys were administered remotely, every six months for four years thereafter with the same web-based forms used at baseline, through Spring 2018 (wave 8). TATAMS human subject's methods were approved by the University of Texas Health Science Center at Houston Institutional Review Board (HSC-SPH-13-0377). Active consent from parents/guardians and assent from students were obtained for all surveys. The retention rates for waves $1-8$ ranged $64-85 \%$. Further description about TATAMS sampling methodology and study design are provided elsewhere ${ }^{23}$. The present study prospectively examined data at baseline (wave 1) and the followup at 4 years (wave 8 ). The analysis sample for this study was limited to adolescents who were in the 10th grade at baseline $(n=1463$; average age $=15.0$ years, $\mathrm{SD}=0.79$ ) with complete data on sociodemographic and risk variables at follow-up $(\mathrm{n}=948$; average age $=18.3$ years, $\mathrm{SD}=0.77$ ). We focused on these older adolescents because they are in a critical period of development; their behavioral decisions during the transition into young adulthood strongly affects their trajectories and have long-term consequences for their health and wellbeing ${ }^{24}$.

\section{Measures}

\section{Dependent variables}

The main outcomes in this analysis were assessed at follow-up in 2018. Past 30-day cigarette use was assessed using the question: 'During the past 30 days, did you smoke a cigarette?'. Response options were 'yes' and 'no.' Participants who responded 'no' at follow-up were classified as non past 30-day cigarette users, participants who responded 'yes' were classified as past 30-day cigarette users. Past 30-day e-cigarette use was assessed using the question: 'During the past 30 days, did you use an electronic cigarette, vape pen or e-hookah?'. Response options were 'yes' and 'no.' Participants who responded 'no' at follow-up were classified as non past 30-day e-cigarette users, participants who responded 'yes' were classified as past 30-day e-cigarette users.

\section{Independent variables}

The main exposure variables were assessed at baseline in 2014. The Strengths and Difficulties Questionnaire (SDQ) is a five-scale mental health screening tool used in children and adolescents ${ }^{21}$. The scales measure emotional symptoms, conduct-problems, hyperactivity-inattention, peer relationship problems, and prosocial behaviors ${ }^{25}$. In the current analysis, we used the emotional symptoms subscale from the SDQ. The emotional symptoms subscale consists of five items that probe somatic symptoms (i.e. 'I get a lot of headaches, stomach aches or sickness' and 'I have many fears, I am easily scared') as well as mental health symptoms (i.e. 'I worry a lot', 'I am unhappy, depressed or fearful', and 'I am nervous in new situations'). Response options include: 'Not true', 'Somewhat true', and 'Certainly true'. Participants who completed at least three of the five items were included in the analyses; a mean composite score was created corresponding to the sum of the items divided by the total number of items completed. Emotional symptoms scores ranged 1-10, with a mean of 2.67 $(\mathrm{SD}=2.73$ ), with higher scores reflecting higher levels of emotional symptoms. Emotional symptoms were divided into three groups based on the distribution: low $(\leq 1)$; medium (2-3); and high (8-14). Based on data from participants in the current analysis, the SDQ demonstrated excellent internal reliability (Cronbach's a=0.96).

We used the Brief Sensation Seeking Scale (BSSS$4 ; 22)$ to assess sensation seeking ${ }^{26}$. The BSSS-4 consists of four items that use a five-point response option, ranging from 'strongly disagree' to 'strongly agree': 1) 'I would like to explore strange places'; 
2) 'I like to do frightening things'; 3 ) 'I like new and exciting experiences, even if I have to break the rules'; and 4) 'I prefer friends who are exciting and unpredictable'. Participants who completed at least two of the four items were included in the analyses; a mean sensation seeking score was created by summing responses across all completed items and dividing by the number of completed items. Sensation seeking scores ranged from 1 to 5 , with a mean of 3.09 ( $\mathrm{SD}=1.04$ ), with higher scores reflecting higher levels of sensation seeking. Sensation seeking symptoms were divided into tertiles based on their distribution: low ( $\leq 2.75)$; medium (>2.75 to 3.5$)$; and high $(>3.5)$. Based on data from participants in the current report, the BSSS-4 demonstrated excellent internal reliability (Cronbach's a=0.95).

\section{Covariates}

Several covariates were included in the analysis to adjust for confounding influences ${ }^{18}$. These covariates included sociodemographic variables: sex (female, male), race/ethnicity (non-Hispanic White, Hispanic, non-Hispanic Black or African American, and non-Hispanic other), age in years, and type of school attended (charter, private, or public). Ever use of either product at baseline also was included in the analysis to control for previous behavior. Ever cigarette use was assessed using the question: 'Have you EVER tried cigarette smoking, even one or two puffs?'. Response options were 'yes' and 'no.' Participants who responded 'no' at baseline were classified as never users; participants who responded 'yes' were classified as ever users. Ever e-cigarette use was assessed using the question: 'Have you EVER used an electronic cigarette, vape pen, or e-hookah, even one or two puffs?'. Response options were 'yes' and 'no'. Participants who responded 'no' at baseline were classified as never users; participants who responded 'yes' were classified as ever users.

\section{Statistical analysis}

Descriptive statistics were calculated as percentages for categorical variables and means and standard deviations for continuous variables. We used a mixedeffects logistic regression to model the association between the exposure variables at baseline (i.e. emotional symptoms, sensation seeking) and the odds of past 30-day cigarette and e-cigarette use at follow- up at 4 years, after adjusting for sociodemographic factors and ever tobacco use at baseline. The random effect was included in the mixed-effects logistic regression to account for the correlation of subjects in the same school. To meet the linearity assumption between the log odds of the outcome and each predictor and to improve the accuracy of our models, we categorized emotional symptoms and sensation seeking variables into tertiles (low, medium, high). A potential interaction between emotional symptoms and sensation seeking was examined to assess whether one modifies the effect of the other on cigarette and e-cigarette use. We included the product term of sensation seeking with emotional symptoms and compared the model with and without the product terms, in terms of model fit using the likelihood ratio test. Stratified analyses were conducted as the interaction was significant. Participants with missing values for any covariates were excluded in the model ( $35 \%$ of the sample). An attrition analysis was conducted to identify differences between participants who provided data at both time points compared to those who provided data at baseline only. Data were expressed as odds ratios (ORs) with 95\% confidence intervals (95\% CIs). Alpha was set at 0.05 and all analyses were conducted using STATA statistical software (version 16.0;23) ${ }^{27}$.

\section{RESULTS}

\section{Sample characteristics}

The sociodemographic characteristics of the 1463 adolescents in 10th grade included in the current analysis are presented in Table 1 . The majority were females (55\%). Approximately $40 \%$ identified as Hispanic, $27 \%$ as non-Hispanic White, $20 \%$ as non-Hispanic Black or African American, and the remaining $14 \%$ identified as non-Hispanic other. Most (82\%) attended public schools. Most (44\%) of the adolescents reported low emotional symptoms, $24 \%$ reported medium emotional symptoms, and $32 \%$ reported high emotional symptoms. Roughly a third (34\%) of the adolescents reported low level of sensation seeking, $31 \%$ reported medium level of sensation seeking, and $32 \%$ reported high level of sensation seeking. In 2014, at baseline, almost $16 \%$ of the 10th graders reported that they had tried cigarettes and $32 \%$ reported they had tried e-cigarettes. By the follow-up at four years in $2018,23 \%$ reported they 
Table 1. Descriptive statistics of the study sample at baseline, TATAMS 2014 ( $\mathrm{N}=1463)$

\begin{tabular}{|c|c|c|}
\hline Characteristics & $n$ & $\%$ \\
\hline \multicolumn{3}{|l|}{ Sex } \\
\hline Female & 807 & 55.16 \\
\hline Male & 656 & 44.84 \\
\hline Age (years), mean, SD & 15.03 & 0.79 \\
\hline \multicolumn{3}{|l|}{ Race/ethnicity } \\
\hline Non-Hispanic White & 401 & 27.41 \\
\hline Non-Hispanic Black & 291 & 19.89 \\
\hline Hispanic & 569 & 38.89 \\
\hline Non-Hispanic Other ${ }^{\mathrm{a}}$ & 202 & 13.81 \\
\hline \multicolumn{3}{|l|}{ School type } \\
\hline Charter & 91 & 6.22 \\
\hline Private & 166 & 11.35 \\
\hline Public & 1206 & 82.43 \\
\hline \multicolumn{3}{|l|}{ Ever cigarette use $\mathrm{e}^{*}$} \\
\hline No & 1228 & 83.94 \\
\hline Yes & 233 & 15.93 \\
\hline \multicolumn{3}{|l|}{ Ever e-cigarette use ${ }^{*}$} \\
\hline No & 995 & 68.01 \\
\hline Yes & 466 & 31.85 \\
\hline \multicolumn{3}{|l|}{ Emotional symptoms**b } \\
\hline Low & 619 & 43.84 \\
\hline Medium & 336 & 23.80 \\
\hline High & 457 & 32.37 \\
\hline Scale, mean, SD & 2.67 & 2.70 \\
\hline \multicolumn{3}{|l|}{ Sensation seeking ${ }^{* * *_{c}}$} \\
\hline Low & 503 & 34.38 \\
\hline Medium & 460 & 31.44 \\
\hline High & 464 & 31.72 \\
\hline Scale, mean, SD & 3.09 & 1.04 \\
\hline
\end{tabular}

had tried cigarettes and $4.5 \%$ reported past 30 -day use, while $36 \%$ reported they had tried e-cigarettes and $9 \%$ reported past 30 -day use (data not shown).

\section{Direct effects: Emotional symptoms}

In Table 2 we present the unadjusted and adjusted associations between emotional symptoms assessed at baseline and past 30-day cigarette and e-cigarette use at follow-up. After adjusting for age, sex, ethnicity, school type, and ever use of cigarettes or e-cigarettes, adolescents with high emotional symptoms had significantly higher odds of reporting past 30-day cigarette and e-cigarette use compared to adolescents with low emotional symptoms (AOR $=1.97$; 95\% CI: $1.07-3.60$, and AOR $=1.68$; 95\% CI: 1.06-2.66), respectively.

\section{Direct effects: Sensation seeking}

In Table 2 we present unadjusted and adjusted associations between sensation seeking assessed at baseline and past 30-day cigarette and e-cigarette use assessed at follow-up. After adjusting for age, sex, ethnicity, school type, and ever use of cigarettes or e-cigarettes, the odds of past 30-day cigarette and e-cigarette use were significantly higher among adolescents with high sensation seeking tendencies compared to adolescents with low sensation seeking tendencies $(\mathrm{AOR}=2.05 ; 95 \% \mathrm{CI}: 1.03-4.10$, and $\mathrm{AOR}=1.68 ; 95 \%$ CI: 1.02-2.76, respectively).

\section{Effect modification: Emotional symptoms interaction with sensation seeking}

The interaction between emotional symptoms and

Table 2. Longitudinal association between emotional symptoms at baseline, sensation seeking at baseline, and cigarette and e-cigarette use at follow-up at four years, among Texas adolescents, TATAMS 2014-2018

\begin{tabular}{|c|c|c|c|c|}
\hline & \multicolumn{2}{|c|}{ Cigarettes $^{a}$} & \multicolumn{2}{|c|}{ E-cigarettes ${ }^{b}$} \\
\hline & $\begin{array}{c}\text { OR }(95 \% \text { CI }) \\
(\mathrm{n}=949)\end{array}$ & $\begin{array}{c}\operatorname{AOR}^{\mathrm{e}}(95 \% \mathrm{CI}) \\
(\mathrm{n}=948)\end{array}$ & $\begin{array}{c}\text { OR }(95 \% \text { CI }) \\
(n=949)\end{array}$ & $\begin{array}{c}\mathrm{AOR}^{e}(95 \% \mathrm{CI}) \\
(n=948)\end{array}$ \\
\hline \multicolumn{5}{|c|}{ Emotional symptoms ${ }^{c}$} \\
\hline Low & Ref. & Ref. & Ref. & Ref. \\
\hline Medium & $0.58(0.27-1.26)$ & $0.64(0.28-1.43)$ & $0.83(0.49-1.39)$ & $0.95(0.55-1.65)$ \\
\hline High & $1.57(0.91-2.70)$ & $1.97(1.07-3.60)$ & $1.46(0.96-2.22)$ & $1.68(1.06-2.66)$ \\
\hline \multicolumn{5}{|c|}{ Sensation seeking ${ }^{\mathrm{d}}$} \\
\hline Low & Ref. & Ref. & Ref. & Ref. \\
\hline Medium & 1.77 (0.88-3.58) & $1.47(0.71-3.04)$ & $1.43(0.88-2.35)$ & $1.28(0.77-2.13)$ \\
\hline High & $2.58(1.32-5.02)$ & $2.05(1.03-4.10)$ & $1.92(1.20-3.09)$ & $1.68(1.02-2.76)$ \\
\hline
\end{tabular}

AOR: adjusted odds ratio. $\mathrm{Cl}$ : confidence interval. Ref.: reference. Bold indicates statistical significance, $p<0.05$. a Current cigarette use indicates self-reported as smoking cigarette(s) in the past 30 days. b Current e-cigarette use indicates self-reported use of electronic cigarette, vape pen or e-hookah in the past 30 days. c Score: Iow $(\leq 1)$; medium (2-3); high (4-10). d Score: low ( $\leq 2.75)$; medium ( $>2.75$ to 3.5); high (>3.5). e Adjusted for sex, age, ethnicity, school type and ever product use at wave 1. 
sensation seeking in regard to their relationship with past 30-day e-cigarette use was statistically significant $(\mathrm{p}=0.01)$, but it was not with past 30-day cigarette smoking $(\mathrm{p}>0.05)$. Results of the analysis stratified by sensation seeking level for past 30-day e-cigarette use are reported in Table 3. Among low sensation seekers, adolescents who reported high levels of emotional symptoms were at increased risk for e-cigarette use at follow-up (AOR=3.43; 95\% CI: 1.38-8.51). However, among adolescents with medium or high levels of sensation seeking, this association was not statistically significant. Results of the analysis stratified by emotional symptom level for past 30-day e-cigarette use are reported in Table 4. Among adolescents with low emotional symptoms, those who reported high levels of sensation seeking were at increased risk for e-cigarette use at follow-up ( $\mathrm{AOR}=3.50 ; 95 \%$ CI: 1.54-7.91). However, among adolescents with medium or high levels of emotional symptoms this association was not statistically significant.

\section{DISCUSSION}

In the current study, we examined whether emotional symptoms result in additional risk for tobacco use among a sample of adolescent Texans with varying levels of sensation seeking - and whether sensation seeking results in additional risk for tobacco use among adolescents with varying levels of emotional symptoms. Using a prospective study design, we found the association between emotional symptoms and e-cigarette use differed depending on the level of sensation seeking: among low sensation seeking youth, those with high levels of emotional problems were at increased risk for e-cigarette use, however, this relationship was not present among adolescents with medium or high levels of sensation seeking. We further found that among youth with few emotional symptoms, those with high levels of sensation seeking were at increased risk for e-cigarette use. This is the first study that has specifically investigated the joint effect of emotional symptoms and sensation seeking

Table 3. Longitudinal association between emotional symptoms assessed at baseline and e-cigarette use, stratified by level of sensation seeking at follow-up at four years, among Texas adolescents, TATAMS 2014$2018(\mathrm{~N}=948)$

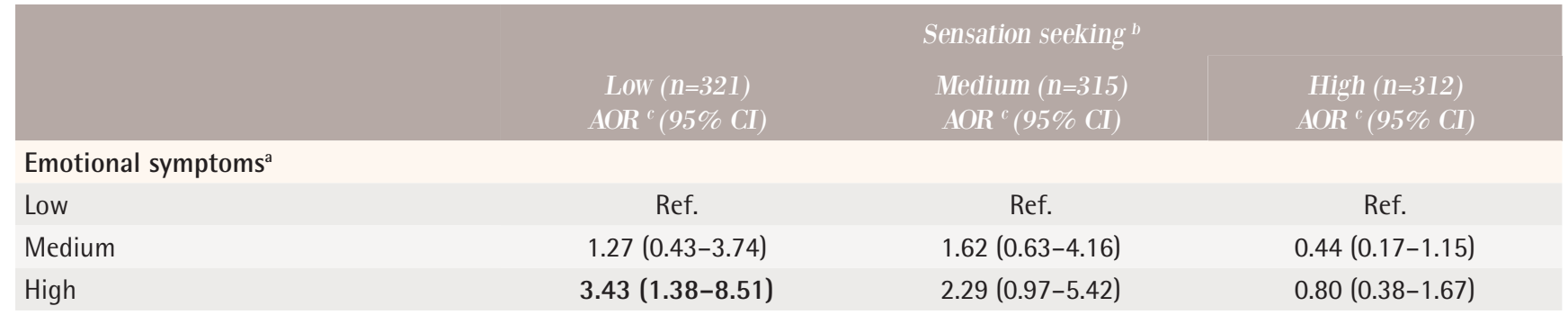

AOR: adjusted odds ratio. Cl: confidence interval. Ref.: reference. Bold indicates statistical significance, $p<0.05$. a Score: low $(\leq 1)$; medium $(2-3)$; high $(4-10)$. b Score: Iow $(\leq 2.75)$; medium ( $>2.75$ to 3.5$)$; high ( $>3.5$ ). c Adjusted for age, sex, ethnicity, school type and ever e-cigarette use. For sensation seeking interaction $p=0.01$.

Table 4. Longitudinal association between sensation seeking assessed at baseline and e-cigarette use at followup at four years, stratified by level of emotional symptoms among Texas adolescents, TATAMS 2014-2018 $(\mathrm{N}=948)$

Emotional Symptoms

Low $(n=321)$

$\operatorname{AOR}^{c}(95 \% \mathrm{CI})$

Medium $(n=315)$ $\operatorname{AOR}^{c}(95 \% \mathrm{CI})$

$\operatorname{High}(n=312)$ $\operatorname{AOR}^{c}(95 \% \mathrm{CI})$

\section{Sensation seeking ${ }^{\mathrm{b}}$}

Low

Ref.

Medium

High
1.27 (0.50-3.20)

3.50 (1.54-7.91)
Ref.

$1.50(0.50-4.47)$

$1.04(0.30-3.58)$
Ref.

0.94 (0.43-2.08)

0.87 (0.40-1.89)

AOR: adjusted odds ratio. Cl: confidence interval. Ref.: reference. Bold indicates statistical significance, $p<0.05$. a Score: low $(\leq 1)$; medium $(2-3)$; high $(4-10)$. b Score: low $(\leq 2.75)$; medium ( $>2.75$ to 3.5$)$; high ( $>3.5$ ). c Adjusted for age, sex, ethnicity, school type and ever e-cigarette use. For emotional symptoms interaction $p=0.01$. 
among adolescents; accordingly, the interactions cannot be directly compared with any existing literature. Although these results do not support our hypothesis, lending support to our novel finding, and consistent with previous literature, we also found that among 10th graders, experiencing emotional symptoms and higher levels of sensation seeking ${ }^{17-19}$ were directly and independently associated with past 30 -day cigarette and e-cigarette use ${ }^{12-15}$, four years after baseline.

As expected and consistent with prior research ${ }^{28}$, adolescents reporting high emotional symptoms in 10th grade were at increased risk of past 30-day cigarette and e-cigarette use four years later; this effect remained after controlling for covariates, including sex, race/ethnicity, age, school type, and ever tobacco use. For example, a Greek study among 1030 participants found that emotional symptoms were positively associated with adolescents' past 30-day cigarette smoking ${ }^{28}$. Research suggests that emotional symptoms and tobacco use are linked in a bidirectional manner: on the one hand, emotional symptoms, such as depression and anxiety, may precede and promote tobacco use but on the other hand, chronic tobacco users may either develop emotional symptoms or worsen their condition ${ }^{29-32}$. Several hypotheses have been proposed regarding the nature of this relationship. The self-medication hypothesis suggests that depressed or anxious people smoke because it is the easiest, most accessible way to control their emotional symptoms, and that leads to smoking initiation and subsequent regular use ${ }^{33}$. Tobacco companies funded research in support of this hypothesis ${ }^{34}$. Alternatively smoking and exposure to nicotine may lead to emotional symptoms by affecting an individual's neurocircuitry, which increases susceptibility to environmental stressors $^{35,36}$. Of note, individuals with emotional symptoms tend to start using tobacco at an earlier age, use it more heavily, and may be less likely to quit $^{37}$ than peers with fewer emotional symptoms. Together these results suggest that developing interventions to address emotional symptoms, as an important risk factor among high school adolescents, may serve to reduce the onset and amount of cigarette and e-cigarette use in the future.

Some adolescents with high sensation seeking tendencies meet their sensation seeking needs through engaging in arousal behaviors that enhance health, rather than behaviors that compromise their health ${ }^{22}$. Consistent with previous research ${ }^{17-19}$, we found that self-reported sensation seeking tendencies reported in 10th grade were associated with product use at follow-up. Our result extends previous research, as the follow-up period in this study was four years. During follow-up many of the participants transitioned to young adulthood, passing the age where they could both legally use tobacco and serve as targets of tobacco marketing ${ }^{38}$, and when tobacco use is often consolidated ${ }^{11,39}$. As such, developing interventions that target adolescents with high sensation seeking tendencies may be a useful strategy. Changing attitudes and beliefs about how to meet arousal needs may lead to the adoption of healthy behaviors rather than health compromising behaviors or the adoption of both healthy behaviors and health compromising behaviors.

The interaction between emotional symptoms and sensation seeking was only observed for e-cigarettes. The odds ratios for the interactions are both statistically significant, both are in the same direction, and both are roughly double those for the direct effects. Thus, in terms of implications for interventions, among youth with low emotional symptoms, the subset of individuals with high sensation seeking tendencies are at increased risk for product use due to their sensation seeking tendencies, while among youth with low sensation seeking tendencies, the subset with high emotional symptoms are at increased risk for product use due to emotional symptoms. As noted, the interaction reveals that the risk for e-cigarette use is almost double among the two subsets of youth. Therefore, identifying these high-risk youth and addressing the reasons for their e-cigarette use may serve as a useful intervention. For example, some youth believe that using tobacco and nicotine will relieve stress ${ }^{8,40}$ and improve their social standing with their peers ${ }^{41}$. Identifying similar beliefs in youth with emotional symptoms and sensation seeking tendencies and providing options and resources to address such beliefs may serve to improve the efficacy of interventions to prevent and reduce product use.

\section{Strengths and limitations}

The current findings should be interpreted within 
the context of study limitations. First, the overall drop-out rate was $35 \%$ and the analysis is restricted to individuals with complete data only; results of such analyses can be biased. We observed some attrition bias over the four years - more males and youth with high sensation seeking tendencies, than females and low sensation seekers, dropped out of the study, which may have underestimated the strength of the associations between sensation seeking and product use, especially as males in general tend to be higher sensation seekers than females ${ }^{17}$. Of note, the overall drop-out rate is similar to that reported from other adolescent Texas-based cohort studies ${ }^{42}$. Second, all items were measured via self-report, therefore, social desirability bias could affect youth responses and cause underreporting of their tobacco use, emotional symptoms and sensation seeking tendencies. Studies should attempt to replicate these results using, for instance, biological measures of tobacco use such as urine or hair screening analysis. Third, risk factors were assessed only at baseline, so it is possible for some risk factors to change across study waves. Fourth, our sample is limited to adolescents residing in the four largest cities in Texas and thus our findings may not be generalizable to other populations. Finally, it is possible that factors other than the variables included in the statistical analyses are related to the associations between emotional symptoms and tobacco use. For example, genetic factors, parents' smoking, and personality type could mediate or modify the abovementioned relationship.

In spite of these limitations, the current study has several strengths including its complex, longitudinal design. Accordingly, we avoid temporal ambiguity in our assessment of adolescent's transition from non-tobacco users to tobacco users. Also, the study utilized well-validated measures of emotional symptoms and sensation seeking that have been used in previous studies including adolescents. Moreover, the current study utilizes an ethnically diverse population-based sample, which is about 40\% Hispanic.

\section{CONCLUSIONS}

The study results confirm previous research and expand our understanding of the role of emotional symptoms and sensation seeking factors on adolescent tobacco risk, with implications for the development of prevention programs among adolescents. It is important for prevention programs to consider both types of predictors in an integrative way and to address both factors since they interact to increase risk of product use more than the sum of emotional symptoms or sensation seeking alone. Therefore, addressing behavioral risk factors, such as sensation seeking, along with emotional symptoms, may serve to increase the efficacy of existing effective intervention strategies in the school environment ${ }^{43}$ in order to curb nicotine and tobacco use among youth.

\section{REFERENCES}

1. World Health Organization. WHO Report on the Global Tobacco Epidemic, 2011: Warning about the dangers of tobacco. World Health Organization; 2011. Accessed January 8, 2021. http://whqlibdoc.who.int/ publications/2011/9789240687813_eng.pdf

2. U.S. Department of Health and Human Services. Preventing Tobacco Use Among Youth and Young Adults: A Report of the Surgeon General. U.S. Department of Health and Human Services, Centers for Disease Control and Prevention, National Center for Chronic Disease Prevention and Health Promotion, Office on Smoking and Health; 2012. Accessed January 8, 2021. https://www. ncbi.nlm.nih.gov/books/NBK99237/pdf/Bookshelf_ NBK99237.pdf

3. Gentzke AS, Creamer M, Cullen KA, et al. Vital Signs: Tobacco Product Use Among Middle and High School Students United States, 2011-2018. MMWR Morb Mortal Wkly Rep. 2019;68(6):157-164. doi:10.15585/mmwr.mm6806e1

4. Niaura R. E-Cigarette Science and Its Relevance for Regulatory Actions and Policy. Nicotine Tob Res. 2018;20(8):911. doi:10.1093/ntr/nty091

5. U.S. Department of Health and Human Services. The Health Consequences of Smoking: 50 Years of Progress. A Report of the Surgeon General. U.S. Department of Health and Human Services, Centers for Disease Control and Prevention, National Center for Chronic Disease Prevention and Health Promotion, Office on Smoking and Health; 2014. Accessed January 8, 2021. https:// www.ncbi.nlm.nih.gov/books/n/surgsmoke50/pdf/

6. U.S. Department of Health and Human Services. E-Cigarette Use Among Youth and Young Adults. A Report of the Surgeon General. U.S. Department of Health and Human Services, Centers for Disease Control and Prevention, National Center for Chronic Disease Prevention and Health Promotion, Office on Smoking and Health; 2016. Accessed January 8, 2021. https://www. ncbi.nlm.nih.gov/books/NBK538680/pdf/Bookshelf_ NBK538680.pdf

7. National Research Council and Institute of Medicine. Preventing Mental, Emotional, and Behavioral Disorders 
Among Young People: Progress and Possibilities. O'Connell ME, Boat T, Warner KE, eds. The National Academies Press; 2009. Accessed February 9, 2021. https://www.ncbi.nlm.nih.gov/books/NBK32775/pdf/ Bookshelf_NBK32775.pdf

8. Kassel JD, Stroud LR, Paronis CA. Smoking, stress, and negative affect: correlation, causation, and context across stages of smoking. Psychol Bull. 2003;129(2):270-304. doi:10.1037/0033-2909.129.2.270

9. Salín-Pascual RJ, Drucker-Colín R. A novel effect of nicotine on mood and sleep in major depression. Neuroreport. 1998;9(1):57-60. doi:10.1097/00001756-199801050-00012

10. Baker TB, Brandon TH, Chassin L. Motivational influences on cigarette smoking. Annu Rev Psychol. 2004;55(1):463491. doi:10.1146/annurev.psych.55.090902.142054

11. Wetter DW, Kenford SL, Welsch SK, et al. Prevalence and predictors of transitions in smoking behavior among college students. Health Psychol. 2004;23(2):168-177. doi:10.1037/0278-6133.23.2.168

12. Patton GC, Carlin JB, Coffey C, Wolfe R, Hibbert M, Bowes G. Depression, anxiety, and smoking initiation: a prospective study over 3 years. Am J Public Health. 1998;88(10):1518-1522. doi:10.2105/ajph.88.10.1518

13. Elkins IJ, McGue M, Iacono WG. Prospective effects of attentiondeficit/hyperactivity disorder, conduct disorder, and sex on adolescent substance use and abuse. Arch Gen Psychiatry. 2007;64(10):1145-1152. doi:10.1001/archpsyc.64.10.1145

14. Kim JS, Kim K. Electronic cigarette use and suicidal behaviors among adolescents. J Public Health (Oxf). 2019:fdz086. doi:10.1093/pubmed/fdz086

15. Fergusson DM, Goodwin RD, Horwood LJ. Major depression and cigarette smoking: results of a 21-year longitudinal study. Psychol Med. 2003;33(8):1357-1367. doi:10.1017/s0033291703008596

16. Zuckerman M. Behavioral expressions and biosocial bases of sensation seeking. Cambridge University Press; 1994.

17. Wilkinson AV, Okeke NL, Springer AE, et al. Experimenting with cigarettes and physical activity among Mexican origin youth: a cross sectional analysis of the interdependent associations among sensation seeking, acculturation, and gender. BMC Public Health. 2012;12:332. doi:10.1186/14712458-12-332

18. Case KR, Harrell MB, Pérez A, et al. The relationships between sensation seeking and a spectrum of e-cigarette use behaviors: Cross-sectional and longitudinal analyses specific to Texas adolescents. Addict Behav. 2017;73:151157. doi:10.1016/j.addbeh.2017.05.007

19. Lipkus IM, Barefoot JC, Williams RB, Siegler IC. Personality measures as predictors of smoking initiation and cessation in the UNC Alumni Heart Study. Health Psychol. 1994;13(2):149-155. doi:10.1037//0278-6133.13.2.149

20. Trujillo Á, Obando D, Trujillo CA. Family dynamics and alcohol and marijuana use among adolescents: The mediating role of negative emotional symptoms and sensation seeking. Addict Behav. 2016;62:99-107. doi:10.1016/j.addbeh.2016.06.020

21. Lynne-Landsman SD, Graber JA, Nichols TR, Botvin GJ. Is sensation seeking a stable trait or does it change over time? J Youth Adolesc. 2011;40(1):48-58. doi:10.1007/s10964-010-9529-2

22. Mckay S, Skues JL, Williams BJ. With risk may come reward: Sensation seeking supports resilience through effective coping. Pers Individ Dif. 2018;121:100-105. doi:10.1016/j.paid.2017.09.030

23. Pérez A, Harrell MB, Malkani RI, et al. Texas Adolescent Tobacco and Marketing Surveillance System's Design. Tob Regul Sci. 2017;3(2):151-167. doi:10.18001/TRS.3.2.3

24. Frech A. Healthy Behavior Trajectories between Adolescence and Young Adulthood. Adv Life Course Res. 2012;17(2):59-68. doi:10.1016/j.alcr.2012.01.003

25. Muris P, Meesters C, van den Berg F. The Strengths and Difficulties Questionnaire (SDQ)--further evidence for its reliability and validity in a community sample of Dutch children and adolescents. Eur Child Adolesc Psychiatry. 2003;12(1):1-8. doi:10.1007/s00787-003-0298-2

26. Stephenson MT, Hoyle RH, Palmgreen P, Slater MD. Brief measures of sensation seeking for screening and largescale surveys. Drug Alcohol Depend. 2003;72(3):279786. doi:10.1016/j.drugalcdep.2003.08.003

27. Stata 16. Version 16. StataCorp; 2019. Accessed January 8, 2021. https://www.stata.com/why-use-stata/

28. Giannakopoulos G, Tzavara G, Dimitrakaki C, Kolaitis G, Rotsika V, Tountas Y. Emotional, behavioural problems and cigarette smoking in adolescence: findings of a Greek cross-sectional study. BMC Public Health. 2010;10:57. doi:10.1186/1471-2458-10-57

29. Breslau N, Peterson EL, Schultz LR, Chilcoat HD, Andreski P. Major depression and stages of smoking. A longitudinal investigation. Arch Gen Psychiatry. 1998;55(2):161-166. doi:10.1001/archpsyc.55.2.161

30. Mojtabai R, Crum RM. Cigarette smoking and onset of mood and anxiety disorders. Am J Public Health. 2013;103(9):1656-1665. doi:10.2105/AJPH.2012.300911

31. Marsden DG, Loukas A, Chen B, Perry CL, Wilkinson AV. Associations between frequency of cigarette and alternative tobacco product use and depressive symptoms: A longitudinal study of young adults. Addict Behav. 2019;99:106078. doi:10.1016/j.addbeh.2019.106078

32. Bandiera FC, Loukas A, Li X, Wilkinson AV, Perry CL. Depressive Symptoms Predict Current E-Cigarette Use Among College Students in Texas. Nicotine Tob Res. 2017;19(9):1102-1106. doi:10.1093/ntr/ntx014

33. Markou A, Kosten TR, Koob GF. Neurobiological similarities in depression and drug dependence: a self-medication hypothesis. Neuropsychopharmacology. 1998;18(3):135174. doi:10.1016/S0893-133X(97)00113-9

34. Prochaska JJ, Hall SM, Bero LA. Tobacco use among individuals with schizophrenia: what role has the tobacco industry played? Schizophr Bull. 2008;34(3):555-567. doi:10.1093/schbul/sbm117 
35. Fluharty M, Taylor AE, Grabski M, Munafò MR. The Association of Cigarette Smoking With Depression and Anxiety: A Systematic Review. Nicotine Tob Res. 2017;19(1):3-13. doi:10.1093/ntr/ntw140

36. Yuan M, Cross SJ, Loughlin SE, Leslie FM. Nicotine and the adolescent brain. J Physiol. 2015;593(16):3397-3412. doi:10.1113/JP270492

37. Lasser K, Boyd JW, Woolhandler S, Himmelstein DU, McCormick D, Bor DH. Smoking and mental illness: A population-based prevalence study. JAMA. 2000;284(20):2606-2610. doi:10.1001/jama.284.20.2606

38. Biener L, Albers AB. Young adults: vulnerable new targets of tobacco marketing. Am J Public Health. 2004;94(2):326-330. doi:10.2105/ajph.94.2.326

39. Clendennen SL, Loukas A, Creamer MR, Pasch KE, Perry CL. Longitudinal Patterns of Multiple Tobacco and Nicotine Product Use Among Texas College Students: a Latent Transition Analysis. Prev Sci. 2019;20(7):10311042. doi:10.1007/s11121-019-01031-3

40. Leventhal AM, Urman R, Barrington-Trimis JL, et al. Perceived stress and poly-tobacco product use across adolescence: Patterns of association and gender differences. J Psychiatr Res. 2017;94:172-179. doi:10.1016/j.jpsychires.2017.07.010

41. Wilkinson AV, Shete S, Vasudevan V, Prokhorov AV, Bondy ML, Spitz MR. Influence of subjective social status on the relationship between positive outcome expectations and experimentation with cigarettes. J Adolesc Health. 2009;44(4):342-348. doi:10.1016/j.jadohealth.2008.08.003

42. Bigman G, Wilkinson AV, Vandewater EA, et al. Viewing images of alcohol use in PG-13-rated movies and alcohol initiation in Mexican-heritage youth. J Ethn Subst Abuse. 2020;19(4):521-536. doi:10.1080/15332640.2018.1548319

43. Flay BR. School-based smoking prevention programs with the promise of long-term effects. Tob Induc Dis. 2009;5(March):1-18. doi:10.1186/1617-9625-5-6

\section{ACKNOWLEDGMENTS}

This work was supported by The National Cancer Institute of NIH (Grant number CA239097) and the Center for Tobacco Products of the US Food and Drug Administration DHHS (Grant number P50 CA180906-01). The content of this manuscript is solely the responsibility of the authors and does not necessarily represent the official views of the NIH or the Food and Drug Administration. NIH/FDA had no role in the study design, collection, analysis or interpretation of the data, writing of the manuscript, or the decision to submit the manuscript for publication.

\section{CONFLICTS OF INTEREST}

The authors have each completed and submitted an ICMJE form for disclosure of potential conflicts of interest. The authors declare that they have no competing interests, financial or otherwise, related to the current work. All authors report grants from The National Cancer Institute of NIH (Grant number CA239097) and the Center for Tobacco Products of the US Food and Drug Administration DHHS (Grant number P50 CA180906-01). M. B. Harrell also reports that she serves as a consultant in litigation involving the vaping industry.

\section{FUNDING}

This work was supported by The National Cancer Institute of NIH (Grant number CA239097) and the Center for Tobacco Products of the US Food and Drug Administration DHHS (Grant number P50 CA180906-01).

\section{ETHICAL APPROVAL AND INFORMED CONSENT}

The TATAMS human subject's methods were approved by the University of Texas Health Science Center at Houston Institutional Review Board (HSC$\mathrm{SPH}-13-0377)$. Active consent from parents/guardians and assent from students were obtained for all surveys.

\section{AUTHORS' CONTRIBUTIONS}

$\mathrm{BB}$ conducted all statistical analyses and wrote the manuscript. MBH served as Principal Investigator for the TATAMS study and contributed to the conceptualization of the manuscript and provided feedback throughout the writing process. KRC, SLC, AS and AVW contributed to the conceptualization of the manuscript and provided critical feedback throughout the writing process. BC provided statistical consultation for the project and serves as statistician for the TATAMS study. All authors contributed to and have approved the final manuscript.

PROVENANCE AND PEER REVIEW

Not commissioned; externally peer reviewed. 